\title{
Bioanalysis
}

\section{Bioanalytical laboratory automation development: why should we and how could we collaborate?}

\begin{abstract}
"..lab automation development collaboration within the bioanalytical industry will benefit every party and is necessary, justifiable and feasible."
\end{abstract}

\begin{abstract}
Keywords: automation $\bullet$ bioanalytical $\bullet$ collaboration $\bullet$ consortium $\bullet$ coopetition $\bullet$ resource sharing $\bullet$ wet-chemistry
\end{abstract}

By now there should have been a consensus [1-3] in the bioanalytical industry that automation in bioanalytical laboratories improves sample throughput and data integrity, shortens method development time [4,5] and sample data turnaround time. In addition, automation reduces tedious labor, occupational hazards, employee turnover and, in general, improves productivity. These factors all lead to benefits in drug development timelines and costing [1-3]. Currently in bioanalysis, the bottleneck is mostly with the wet chemistry/sample preparation portion of the workflow, be it either sample preparation method development or sample preparation for routine analysis [2]. Much like the autosampler made large, automated, overnight LCMS/MS batch runs possible, the best way to improve bioanalytical wet chemistry productivity would be a commercially available, all-in-one 'bioanalytical wet chemistry kiosk' (BWCK) [2,6], which every bioanalytical lab could afford. The BWCK would take some time to become a reality and perfect its functionalities; but even if the BWCK were available today, it would take a long time for it to phase into mainstream use and completely replace the current, large installed base of generic liquid-handling robots in production. As capital equipment, current generic liquid-handling robots will take many years to depreciate their full value.

\section{Automation development: why should we collaborate?}

While we wait for the instrument vendors to come up with the BWCK and for our labs to embrace it, I believe the bioanalytical industry should not wait to collaborate on bioanalytical lab automation development, for the following reasons detailed below.

Only when the whole industry moves towards automation, would automation benefit every party. Currently, large-scale automation in the bioanalytical industry is still the exception rather than the norm and the economy of scale from automation in the industry is too little to discern. Many companies do not have the resources to develop inhouse automation capabilities and many simply outsource bioanalytical work. However, at the destination (domestic) CROs where the work is done, there is no economy of scale from automation either. At emerging market CROs, besides the lack of large-scale automation, the labor cost is also steadily rising, among other things. Therefore, the cost for outsourcing bioanalytical work is not coming down quick enough and the cost is passed back onto the sponsors. Our production metrics tell us that, with highly automated small-molecule sample preparation capabilities [7], our internal small-molecule bioanalysis throughput, data turnaround time and data quality are better than our CROs. That being said, we still outsource some of our bioanalytical work owing to overall capacity limitations, at a higher overall cost of course. It is conceivable that if large-scale automation were in production at all CROs, the CROs would have more efficient operations; outsourcing cost would have come down noticeably, data turnaround would have been faster and data quality better, all

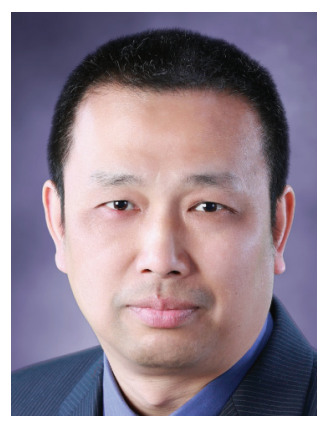

Ming Li

Biogen Idec, Inc., 14 Cambridge Ctr.,

Cambridge, MA 02142, USA

Tel.: +1 6176792922

ming.li@biogenidec.com 
benefiting the sponsors. Thus, automation does need to be an industry-wide phenomenon to benefit all parties involved.

Large-scale bioanalytical automation, as mentioned above, needs to be simple-to-use, mindful of user errors, assay class automation, robust and tied into enterprise IT systems (SMART) [2]. If a large-scale automated bioanalytical lab needed as many automation operators, code developers and/or code maintenance workers and hardware support engineers as a same throughput, traditional bioanalytical lab, then corporations would not see added value for automation and so the arrangement would not be sustainable. In order for companies to reduce labor and operational support costs, SMART automation should be as close to a turn-key automation solution as possible: intuitive and requiring little training to operate and running mostly error-free [2]. SMART automation systems take time to plan, design, develop, test and deploy [8-10]. In general, the bioanalytical industry has a shortage of automation development expertise and, as a result, automation specialists are easily regarded as a flight risk [11]. Therefore, it is often not sustainable for any single company to invest in the training and maintaining of a large troop of automation expertise.

\section{"Owing to the long depreciation cycle of capital equipment, assorted liquid-handling robot platforms are going to be in service for a long time."}

SMART automation research reported in the literature might originate from a few specific robotic platforms, but, in fact, the concept is applicable to all current liquid-handling robots on the market. For example, the output of the same Windows graphical user interface program, which is the culmination of built-in bioanalytical business logic and liquidhandling error prevention algorithm, often contains complete liquid-handling schemes $[4,7]$ that could be executed on different robotic platforms using their corresponding robot scripts. Each robotic platform has its unique features and advantages that might be best suited to particular applications. Within a single company, there might be multiple liquid-handling robotic platforms. Owing to the long depreciation cycle of capital equipment, assorted liquid-handling robot platforms are going to be in service for a long time. On the other hand, it is extremely rare for a single person to be an expert in every liquid-handling robotic platform. Therefore, often times, a company has a handful of automation experts on platform A, but no expert on platform $B$. The situation might be the opposite at another company, which has expertise on platform B but not platform A, and so on and so forth. Pooling and sharing automation development expertise among the whole bioanalytical industry would be the most effective way of fully leveraging all robotic platforms' capabilities. For example, one group of people could focus on the graphical user interface programs for assorted workflows and provide detailed specifications of the output, while other experts could start from the same graphical user interface output files and write different robot execution scripts for their most familiar robotic platforms. By sharing the work, all people using all kinds of robotic platforms would achieve same-quality automation.

\section{"By sharing the work, all people using all kinds of robotic platforms would achieve same-quality automation."}

SMART automation approach is not limited to the automation of all bioanalytical assay types, be they sample preparation for routine analysis [7,12] or sample preparation method development [4,5], as long as microtiter plates associated with those assay types are available [13]. It is also applicable to the automation of many bioanalysis-related workflows, such as some drug screening assays [14], which has implications for high throughput ADME screening assays. The strategy of mapping workflow into a set of inter-related parameters, integrating business logic, applying experimental boundary conditions, adding user input checks and liquid-handling error prevention measures into algorithm, and computing a comprehensive liquid-handling scheme for execution has proven to be so successful through our practices, that it has even enlightened colleagues outside our discipline what SMART automation could do to simplify their lives. As the number of SMART automation systems grows, my team has been stretched so thin to cover everything from workflow mapping, systems analyses, systems development, testing, training, support and maintenance, that the full SMART automation development cycle is getting longer and longer. Other companies might be experiencing the same thing. It definitely cannot continue as such forever and it is necessary to take action to make it sustainable (e.g., industry-wide collaboration).

\section{Automation development: how can we collaborate?}

To speed up bioanalytical lab automation development and implementation throughout the industry, there is a whole spectrum of possible options. At one end of the spectrum would be something similar to the opensource movement in the computer industry, in which computer software source code is made freely available 
to the public. But this idea most likely will not stick, since there is a cost associated with the training and maintenance of lab automation scientists and companies would not give away something for nothing. In addition, it is too loosely structured to have coherence, coordination and support. At the other end of the spectrum would be a for-profit, venture capital-backed start-up. There are two problems with this option. The first is slow start, since it takes time to educate the venture capitalists on what it is and its merits. Second is slow penetration, because it takes time to attract experts on all robotic platforms to come on board, if they are willing to come on board at all, that is. Also, its products' pricing may affect pace of penetration.

Somewhere in the middle of the spectrum lies coopetition backed by a consortium, which I believe is the most promising route. Coopetition, coined from cooperation and competition, refers to otherwise competing companies collaborating in a consortium to cooperate on non-strategic areas of their core businesses, in order to reduce their costs in these areas and compete in other areas where they can differentiate better. Bioanalytical industry-wide lab automation development collaboration fits the coopetition definition squarely. It would pool scarce bioanalytical lab automation development resources, reduce redundant development effort in every company and speed up development, deployment and support of bioanalytical automation systems significantly.

\section{References}

1 Yuan L, Ji QC. Automation in new frontiers of bioanalysis: a key for quality and efficiency. Bioanalysis 4(23), 2759-2762 (2012).

2 Allinson JL, Blick KE, Cohen L, Higton D, Li M. Ask the experts: automation: part I. Bioanalysis 5(16), 1953-1962 (2013).

3 Miller V, Shoup R, Szapacs M, Wang J, Yuan L, Tweed J. Ask the experts: automation: part II. Bioanalysis 5(17), 2085-2094 (2013).

4 Li M, Chou J, King K, Yang. ASPECTS: an automationassisted SPE method development system. Bioanalysis 5(13), 1661-1676 (2013).

5 Singleton C, Li M. Automated sample preparation method development. In: Eliminating Bottlenecks for Efficient Bioanalysis: Practices and Applications in Drug Discovery and Development. Shou WZ, Weng N (Eds). Future Science Ltd, London, UK, 6-17 (2014).

$6 \mathrm{Li} \mathrm{M}$. Automation in the bioanalytical laboratory: what is the future? Bioanalysis 5(23), 2859-2861 (2013).

7 Li M, Chou J, Jing J et al. MARS: bringing the automation of small-molecule bioanalytical sample preparations to a new frontier. Bioanalysis 4(11), 1311-1326 (2012).

\section{Conclusion}

For the reasons outlined above, I strongly believe that lab automation development collaboration within the bioanalytical industry will benefit every party and is necessary, justifiable and feasible. I hope the bioanalytical industry would reach this consensus as well soon, for only then can the discussion of the exact format of the collaboration and implementation details follow. Only then, can we start to speed up the widespread adoption of bioanalytical lab automation. And only then, will we start to get closer to reaping the benefits of bioanalytical lab automation's economy of scale.

\section{Disclaimer}

The opinions and views expressed in this article are solely those of the author and do not necessarily reflect the views of Future Science Ltd.

\section{Financial \& competing interests disclosure}

The author has no relevant affiliations or financial involvement with any organization or entity with a financial interest in or financial conflict with the subject matter or materials discussed in the manuscript. This includes employment, consultancies, honoraria, stock ownership or options, expert testimony, grants or patents received or pending, or royalties.

No writing assistance was utilized in the production of this manuscript.

Shou WZ, Zhang J. Recent development in software and automation tools for high-throughput discovery bioanalysis. Bioanalysis 4(9), 1097-1109 (2012).

9 Zelesky V, Schneider R, Janiszewski J, Zamora I, Ferguson J, Troutman M. Software automation tools for increased throughput metabolic soft-spot identification in early drug discovery. Bioanalysis 5(10), 1165-1179 (2013).

10 Li M, Chou J. Automation tools. In: E-tools in Bioanalysis. Future Science Ltd, London, UK (2014) (In Press).

11 Cohen LH. Surrendering to the robot army: why we resist automation in drug discovery and development. Bioanalysis 4(9), 985-987 (2012).

12 Patel V, Leach D, Hornberger M et al. Automating bioanalytical sample analysis through enhanced system integration. Bioanalysis 5(13), 1649-1659 (2013).

13 Wei D, Li M, King K, Yang L. Online and automated sample extraction. In: Sample Extraction Techniques for Biological Samples: Recent Advances and Novel Applications. Future Science Ltd, London, UK (2014) (In Press).

14 Li M, Chou J, King K, Jing J, Wei D, Yang L. ICECAP: an integrated general purpose automation-assisted IC50/EC50 assay platform. JALA (2015) (In Press). 